\title{
Concurrent task effects on memory encoding and retrieval: Further support for an asymmetry
}

\author{
MOSHE NAVEH-BENJAMIN, ANGELA KILB, and TYLER FISHER \\ University of Missouri, Columbia, Missouri
}

\begin{abstract}
Several studies have demonstrated that divided attention at encoding significantly reduces memory performance, whereas divided attention at retrieval affects memory performance only minimally. However, the possibility exists that retrieval processes have shown such resilience because the concurrent tasks used have often not been very demanding. To assess this possibility, we used independent manipulations of the concurrent task during either encoding or retrieval that included stimulus-response compatibility and participant- versus experimenter-controlled pace. In addition, we manipulated the distribution of practice that the participants received with the primary and the concurrent tasks. The results replicated and extended those recently reported by Rohrer and Pashler (2003), indicating that although memory performance is negatively affected by divided attention at retrieval, especially with noncompatible stimulus-response mapping in the concurrent task, this effect was much smaller than that at encoding, in line with the asymmetry notion. Furthermore, experimenter versus participant control of the concurrent task had no effect on memory retrieval. Finally, under conditions of equal practice with both the memory and the concurrent tasks, memory retrieval was affected only to a small degree. In contrast to encoding processes, the processes involved in retrieval accuracy appear, in many cases, to be less interrupted by divided attention, although this protection requires substantial resources.
\end{abstract}

Memory researchers have shown much interest in the effects of divided attention (DA) on memory performance. Studies assessing these effects have twofold effects: They add to our knowledge of attention and memory processes and of the interplay between them, and they provide an understanding of many situations in which people have to encode or retrieve information under DA conditions. Such conditions may include cases in which a person must retrieve an answer to a question asked by a passenger while carrying out another complex task, such as driving in an urban area, or having to split one's attention between social cues and encoding someone's name when being introduced to a new person. Such occasions, which are characterized by the necessity to attend simultaneously to several sources of information, some of which we have to remember later on, may result in a failure to encode or retrieve the relevant information.

Several studies have indicated that the effects of DA on memory performance depend on whether the dividing of attention happens during the encoding of the information or during its retrieval. For example, Baddeley, Lewis, Eldridge, and Thomson (1984), Craik, Govoni, NavehBenjamin, and Anderson (1996), Johnston, Greenberg,

This research was supported in part by a grant from the Research Board, University of Missouri, Columbia, to the first author. We are grateful to members of the Memory and Cognitive Aging Laboratory at the University of Missouri, Columbia, for their help in conducting the research. Correspondence concerning this article should be addressed to M. NavehBenjamin, Department of Psychological Sciences, University of Missouri, 106 McAlester Hall, Columbia, MO 65211 (e-mail: navehbenjaminm@ missouri.edu).
Fisher, and Martin (1970), Martin (1970), and NavehBenjamin, Craik, Guez, and Dori (1998) have shown that dividing attention during encoding negatively affects both memory and secondary task performance, relative to full-attention conditions. These secondary task costs have been interpreted as reflecting the amount of attentional resources required by encoding processes (Craik, NavehBenjamin, \& Anderson, 1998; Kerr, 1973). In contrast, dividing attention during retrieval has been shown to affect memory performance minimally, although secondary task performance is negatively affected. The conclusion drawn from these results is that retrieval processes are obligatory and resistant to interference but that such resilience requires considerable effort (Craik et al., 1996; NavehBenjamin, Craik, Perretta, \& Tonev, 2000).

There are some occasions on which DA during retrieval significantly affects memory performance, but these seem to be restricted to cases in which source memory is tested (Jacoby, 1991; Troyer, Winocur, Craik, \& Moscovitch, 1999) or to cases in which both the concurrent and the memory tasks involve verbal word form materials (Fernandes \& Moscovitch, 2000, 2002, 2003; Park, Smith, Dudley, \& Lafronza, 1989).

The main purpose of the present study was to assess the degree to which retrieval processes are obligatory and mostly resilient to the effects of DA. One possibility is that the secondary task in the above-mentioned studies was not demanding enough, allowing the participants to retrieve information while performing the secondary task. We wished to evaluate the degree to which retrieval processes show resilience even when the secondary task is made particularly demanding. 
A recent study dealing with the issue of the difficulty of the secondary task and its role in the relative resilience of retrieval processes was carried out by Rohrer and Pashler (2003). They noted that a potential reason for the lack of the effects of DA during retrieval on the free recall task (e.g., Anderson, Craik, \& Naveh-Benjamin, 1998, Experiment 1; Baddeley et al., 1984, Experiment 1; Craik et al., 1996, Experiments 1 and 2; Naveh-Benjamin et al., 1998, Experiment 2) could be the absence of a serious challenge by the competing concurrent task on the centrally demanding stages of memory retrieval. Rohrer and Pashler claimed that when the concurrent task possesses such low temporal density, which is created by the short period of time devoted to the centrally demanding stages of the concurrent task, there may be opportunities to perform the processing operations required by the concurrent task and still be able to protect memory retrieval. In particular, they claimed that in almost all of the aforementioned experiments, a visual four-choice reaction time (RT) concurrent task was used. This task involves an asterisk that appears in one of four spatial boxes within a row, and the participants' task is to respond by pressing the appropriate one of four horizontally adjacent response keys. According to Rohrer and Pashler, this vertical alignment of response keys with the stimulus boxes creates, after some practice, a high stimulus-response compatibility that ensures an easy response selection process that minimizes the centrally demanding aspects of the task (Pashler, 1998). This compatibility therefore allows the response selection process during retrieval to progress with little or no interference. Rohrer and Pashler also suggested the possibility that the use of a self-paced choice in the secondary task contributes to the lack of effect on memory retrieval; the self-pacing used in the above-mentioned studies may have allowed the participants to "cheat" on the concurrent task by giving priority to the memory task, especially when memory retrieval was difficult. This, to their mind, is also the reason why performance on the concurrent task slowed significantly, resulting in the larger concurrent task costs noted in several of the studies mentioned above.

To test these notions, Rohrer and Pashler (2003) used a concurrent task with greater demands on central processes. They used a concurrent task performed simultaneously with a free recall task that was experimenter paced and had low stimulus-response spatial compatibility. Specifically, they used one of three colored stimuli, which appeared randomly in the middle of the screen, and the participants' task was to respond to one of three preassigned keys on the keyboard designated as the response for this color. The key element in using this task was that the identity of the correct response was not compatible with the spatial location of the stimulus. In addition, the pace of the serial choice RT was determined by the experimenter and was presented with a constant interstimulus interval (ISI). The results of Rohrer and Pashler's experiment showed that when performed simultaneously with the concurrent task, free recall performance showed about $25 \%$ reduction in accuracy and slower response latency, relative to performance under full attention. Rohrer and
Pashler interpreted this finding to mean that memory retrieval cannot be performed simultaneously with an unrelated, demanding concurrent task.

Although we believe that the study by Rohrer and Pashler (2003) shows that the type of the secondary task used is important in evaluating the effects of DA on retrieval processes and agree with some of their conclusions, we do have reservations about others, which will be discussed below. In addition, we believe that the methodology employed in their study left several questions unanswered with respect to the asymmetry of the effects of DA on encoding and retrieval processes and, in particular, with respect to the question regarding the resilience of retrieval processes to interference.

First, since they confounded the effect of concurrent task stimulus-response compatibility and pace control by using a noncompatible task that was experimenter paced, we do not know which of these was responsible for the large memory decrements shown. Second, in order to tailor the pace of the concurrent task to individual participants, their practice phase included 10 trials of 1 min each of the concurrent task and only 2 trials of the free recall task, 1 performed alone and 1 under DA. It is possible that this practice regime, which was devoted almost exclusively to the concurrent task, improved the participants' performance on the concurrent task but did not provide them with enough practice with either the free recall task alone or with carrying out both tasks simultaneously. This could have led to the poor memory performance under DA at retrieval.

Third, since Rohrer and Pashler (2003) used the concurrent task manipulation only during retrieval, their results do not directly address the question of symmetry in the effects of DA on encoding and retrieval. In particular, even if retrieval is affected by a concurrent task with high temporal density, such as the one used in their study, such results do not tell us whether the manner in which retrieval is affected by such a task is similar to that in which encoding is affected. This matter is relevant to the question of symmetry, since the claim made in the literature has been as much about the asymmetry in the effects of DA on encoding and retrieval as about the absolute effects of DA at retrieval. For example, Craik et al. (1996) found, in both Experiments 1 and 2, that DA during free recall caused an approximate $10 \%$ reduction in performance, relative to the full-attention condition. In contrast, similar DA during encoding resulted in a much larger decrement of about $50 \%$.

The experiment reported in this article is intended to provide further information on the effects of different features of the concurrent task on encoding and retrieval and, in particular, on the resiliency of the retrieval process to interference. First, in order to determine the conditions under which DA affects free recall performance, we independently manipulated two aspects of the concurrent task: the concurrent task stimulus-response compatibility and the control of the concurrent task pace. We did so by using different trials with all combinations of stimulus-response compatibility and concurrent task pace control. The con- 
current task was an auditory analogue to the one used by Rohrer and Pashler (2003), wherein one of three letters was presented and the participants had to respond by pressing a corresponding key. In the compatible condition, the letters presented were $\mathrm{A}, \mathrm{B}$, and $\mathrm{C}$, with the participants responding on three adjacent keys on the keyboard (V, B, and $\mathrm{N}$, respectively, which were covered). The participants in this condition could use their knowledge of the alphabetical order of the presented letters that corresponded to the left-right horizontal alignment of the response keys to select the appropriate response. In the noncompatible stimulus-response condition, the participants heard the letters $\mathrm{R}, \mathrm{L}$, and $\mathrm{P}$ and responded correspondingly, using the aforementioned keys. Note that in this condition, the order of the correct responses from left to right (R, L, and $\mathrm{P})$ was not compatible with the order of the letters in the alphabet (L, P, and R), as was the case in the compatible condition (see details in the Method section). ${ }^{1}$

We also manipulated the control of the concurrent task pace. In the participant-controlled pace conditions, response to a given concurrent task stimulus triggered the appearance of the next stimulus (as has been done in almost all the studies to date), whereas in the experimentercontrolled pace conditions, the rate of the presentation of the stimuli was fixed (although adjusted during practice to the participants' overall speed; see details in the Method section). This resulted in a $2 \times 2$ factorial design. Note that in addition to the assessment of the main effects and the interaction, this design also allows a direct comparison of the two extreme conditions (compatible, participant paced and noncompatible, experimenter paced), with the former having been used in most of the previous studies mentioned above and the latter having been used in Rohrer and Pashler's (2003) study.

The second question we addressed involved the degree to which different practice regimes, which prioritize the memory or the concurrent task, modulate the effects of DA on retrieval (and encoding). For example, the results reported by Rohrer and Pashler (2003) could have been due to the fact that the practice phase in their study was devoted mostly to the concurrent task. In order to investigate this question, we manipulated the distribution of practice spent on the memory and the concurrent tasks. Half of the participants in the experiment reported received a concurrent task emphasis practice (as used by Rohrer \& Pashler, 2003), whereas the other half received equal amounts of practice in both tasks (see details in the Method section).

Third, in order to assess the degree to which the asymmetry in the effects of DA at encoding and retrieval still held under the different concurrent task conditions, DA in the experiment reported was manipulated during either encoding or retrieval.

In addition to DA conditions, we used baseline control conditions. One such condition was for the memory task, in which the word list was encoded and retrieved under full attention, and the other control condition was for the concurrent task, wherein it was performed alone.

\section{METHOD}

\section{Participants}

The participants were 48 University of Missouri undergraduates, who participated in the experiment in exchange for course credit.

\section{Design}

Four independent variables were used, three of which were manipulated within participants. The first independent variable was attention (full attention, divided attention at encoding, or divided attention at retrieval). The second variable was stimulus-response compatibility (compatible vs. noncompatible). The third variable was concurrent task pace control (participant vs. experimenter paced). The fourth variable, distribution of practice (mostly on the concurrent task vs. equal on both tasks), was manipulated between participants. The dependent variables were proportion of correctly recalled targets in the free recall task, retrieval latency in the free recall task, and performance on the concurrent choice RT (CRT) task

\section{Materials}

The words used were high-frequency monosyllabic words. Nineteen lists were created, with 15 words in each. Each word was used in only one of the lists. The words in each list were not related to each other semantically or in any other obvious way. Sixteen of the lists appeared under DA and were replications of all combinations of concurrent task compatibility, pace control, and locus of divided attention. Three of the lists constituted replications of the full-attention condition. At study, words were presented for $4 \mathrm{sec}$ each. During the test, the participants had $60 \mathrm{sec}$ to recall the words aloud.

The concurrent task involved a sequential auditory presentation, via headsets, of one of three designated letters, presented one at a time, with the participants responding as soon as they could by pressing the appropriate key. In the compatible condition, one of three letters $(\mathrm{A}, \mathrm{B}$, or $\mathrm{C})$ was presented, and the participants had to respond on the adjacent $\mathrm{V}, \mathrm{B}$, or $\mathrm{N}$ key, respectively (V for $\mathrm{A}, \mathrm{B}$ for $\mathrm{B}$, and $\mathrm{N}$ for $\mathrm{C}$ ). To avoid confusion, the original verbal labels on these keys were covered. In the noncompatible condition, one of three letters $(\mathrm{R}, \mathrm{L}$, or $\mathrm{P})$ was presented, and the participants had to respond to the same keys, respectively ( $\mathrm{V}$ for $\mathrm{R}, \mathrm{B}$ for $\mathrm{L}$, and $\mathrm{N}$ for $\mathrm{P}$; see note 1). In addition to having the concurrent tasks performed in the DA conditions, the participants also performed the concurrent tasks alone eight times, two in each of the four combinations of compatibility and control, for $60 \mathrm{sec}$ each.

\section{Procedure}

The participants were run individually. During the practice phase, the participants received practice with the memory task, the concurrent task, and both together (DA). In the equal practice condition, the participants received the same amount of practice with the memory and the concurrent tasks. This included 4 concurrent task trials, 1 in each combination of compatibility and control, 4 trials of full attention, and 4 trials under divided attention ( 2 at encoding and 2 at retrieval). In the concurrent task emphasis practice condition, the participants received 8 concurrent task trials ( 2 in each combination of compatibility and control), 1 full-attention trial, and 2 trials under DA (1 at encoding and 1 at retrieval). This distribution was almost identical to the one used by Rohrer and Pashler (2003), who used 12 trials, with only 1 under DA.

As in Rohrer and Pashler's (2003) study, the ISI for the experimentercontrolled CRT task pace varied between participants and was adapted to the speed of the performance of each participant during the practice phase. On the basis of data obtained from pilot participants, we started with an initial ISI of $1,130 \mathrm{msec}$ (which included $330 \mathrm{msec}$ for stimulus presentation and $800 \mathrm{msec}$ of silence) and adapted the rate throughout the practice phase to each participant's speed of 
response. The ISI used in the experimental trials was equal to the 90th percentile of the participant's CRTs from the last two trials of practice (as in Rohrer \& Pashler, 2003). In the participant-controlled pace condition, the rate was determined by the participants' actual responses, which caused the next stimulus to appear.

After the end of the practice phase, the participants went through the 27 trials, 19 of which were memory trials that included study and test phases and 8 of which were trials that involved performance on the concurrent task alone. For the memory trials, the participants were told to try to learn as many of the words as possible and to recall as many of them as they could during the retrieval phase. For the concurrent task trials, they were told to try to respond as quickly and as accurately as they could to each of the letters presented. In the DA trials, they were told to pay equal attention to both tasks and to try to perform on each to the best of their ability.

The order of the different memory trials was counterbalanced across participants in each of the practice groups, using a Latin square design, and the concurrent baseline trials were inserted in between the memory trials.

In the full-attention trials, the participants studied the 15 words for $4 \mathrm{sec}$ each, and after an interpolated activity of $40 \mathrm{sec}$ (counting three-digit numbers backward, starting with a given number), they tried to orally recall as many of the words as they could for $60 \mathrm{sec}$. Their responses were recorded by the experimenter, who also pressed a key for each recall response on another computer that was synchronized with the presentation computer. The same procedure was used in the DA conditions, except that the participants encoded (or retrieved) the words while performing the concurrent task. The sessions were also tape recorded.

\section{RESULTS}

\section{Recall Task}

The mean number of words recalled correctly (out of 15) across trials and participants for each condition appears in Table 1. This table indicates that performance under full attention was superior to that under the DA conditions and that DA at retrieval resulted in better memory performance than did DA at encoding. The results also show that performance was better in the equal practice condition than in the concurrent task emphasis practice condition and that performance was better in the compatible than in the noncompatible condition. Finally, the results indicate a lack of effect of pace control.

The patterns above were supported by two ANOVAs. The first one, a two-way ANOVA with attention and practice as factors, showed a significant effect of attention
$\left[F(2,92)=194.01, M S_{\mathrm{e}}=0.90, p<.01\right]$. A comparison of full attention $(M=7.75)$ and DA at encoding $(M=$ 3.99) showed a significant difference $[F(1,46)=333.70$, $\left.M S_{\mathrm{e}}=1.03, p<.01\right]$, as did a comparison of full attention and DA at retrieval $\left[M=6.42 ; F(1,46)=57.69, M S_{\mathrm{e}}=\right.$ $0.83, p<.01]$. The comparison of DA at encoding and DA at retrieval was also significant $[F(1,46)=158.16$, $\left.M S_{\mathrm{e}}=0.85, p<.01\right]$. These results replicate those reported in the literature (e.g., Craik et al., 1996; NavehBenjamin et al.,1998; Naveh-Benjamin, Craik, Perretta, \& Tonev, 2000): DA at encoding resulted in a larger decrease in memory performance ( $48 \%$ drop from the full attention condition) than did DA at retrieval (16\%).

The ANOVA also showed a trend for an effect of practice $\left[F(1,46)=2.65, M S_{\mathrm{e}}=4.62, p=.11\right]$, where recall performance was better when equal practice was devoted to the memory and the concurrent CRT tasks $(M=6.34$ and 5.72 for the equal practice and the concurrent task emphasis conditions, respectively). Finally, the interaction of attention and practice was significant $[F(2,92)=3.28$, $\left.M S_{\mathrm{e}}=0.90, p<.05\right]$. The source of this interaction was the superior recall performance in the two DA conditions in the equal practice group over the concurrent task emphasis practice group, coupled with the same recall performance in the two practice groups in the full-attention condition (see Figure 1). These results attest to the success of the practice manipulation, which resulted in superior recall performance when the practice phase was devoted equally to the memory task and the concurrent task than when practice was devoted mostly to the concurrent task.

To enable a full factorial design analysis, the second ANOVA was carried out using the DA conditions only. The means for this analysis are presented in Table 1. A four-way ANOVA was conducted with attention, distribution of practice, compatibility, and pace control as the four independent variables and recall performance as the dependent variable. The analysis showed the effect of attention to be significant $\left[F(1,46)=158.16, M S_{\mathrm{e}}=3.40\right.$, $p<.01]$, where performance was better in the DA-atretrieval condition. The effect of practice was also significant $\left[F(1,46)=6.62, M S_{\mathrm{e}}=10.94, p<.05\right]$, showing that performance improved when the participants received equal amounts of practice with the two tasks. In addition,

Table 1

Means and Standard Deviations for Number of Words Recalled Correctly (Out of 15) in Each of the Conditions

\begin{tabular}{|c|c|c|c|c|c|c|c|c|c|c|}
\hline \multirow{3}{*}{$\begin{array}{l}\text { Stimulus-Response } \\
\text { Mapping }\end{array}$} & & & \multicolumn{4}{|c|}{ Experimenter-Controlled Pace } & \multicolumn{4}{|c|}{ Participant-Controlled Pace } \\
\hline & \multicolumn{2}{|c|}{ Full Attention } & \multicolumn{2}{|c|}{$\begin{array}{l}\text { Divided Atten- } \\
\text { tion at Encoding } \\
\end{array}$} & \multicolumn{2}{|c|}{$\begin{array}{l}\text { Divided Atten- } \\
\text { tion at Retrieval }\end{array}$} & \multicolumn{2}{|c|}{$\begin{array}{l}\text { Divided Atten- } \\
\text { tion at Encoding }\end{array}$} & \multicolumn{2}{|c|}{$\begin{array}{l}\text { Divided Atten- } \\
\text { tion at Retrieval }\end{array}$} \\
\hline & $M$ & $S D$ & $M$ & $S D$ & $M$ & $S D$ & $M$ & $S D$ & $M$ & $S D$ \\
\hline \multicolumn{11}{|c|}{ Equal Emphasis } \\
\hline None & 7.81 & 1.11 & & & & & & & & \\
\hline Compatible & & & 4.86 & 0.94 & 6.94 & 1.45 & 4.06 & 1.15 & 7.02 & 1.81 \\
\hline Noncompatible & & & 4.33 & 1.12 & 6.50 & 1.65 & 4.29 & 1.13 & 6.77 & 1.98 \\
\hline \multicolumn{11}{|c|}{ Concurrent Task Emphasis } \\
\hline None & 7.69 & 2.10 & & & & & & & & \\
\hline Compatible & & & 3.81 & 1.89 & 6.10 & 1.58 & 3.68 & 1.88 & 6.23 & 2.04 \\
\hline Noncompatible & & & 3.18 & 1.61 & 5.52 & 1.67 & 3.60 & 1.52 & 5.69 & 2.53 \\
\hline
\end{tabular}




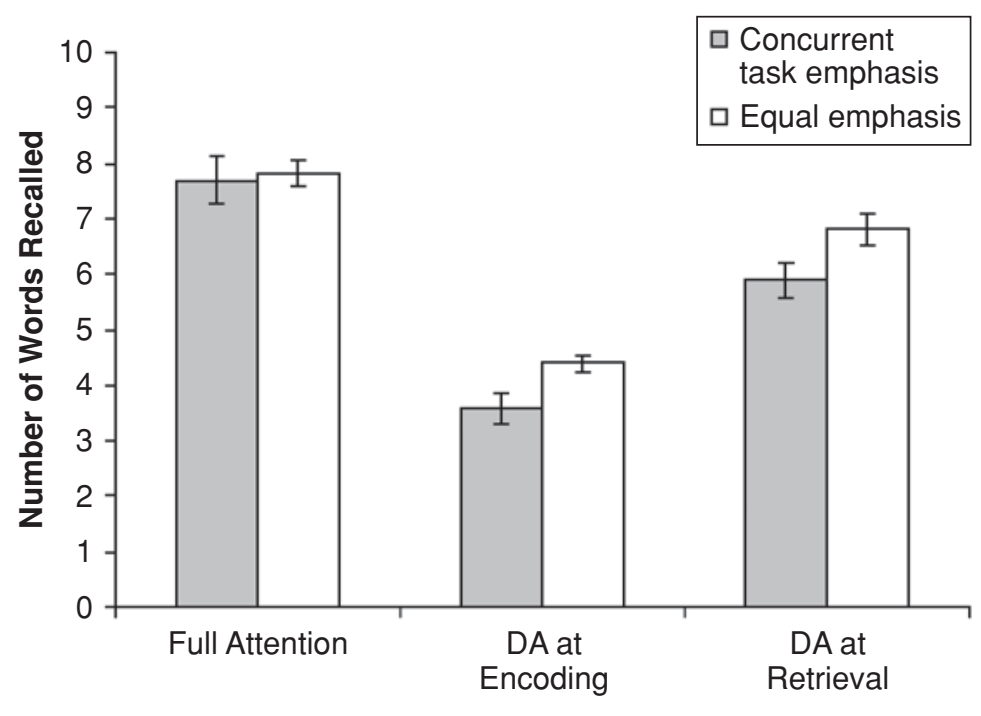

Attention

Figure 1. Recall performance ( $\pm S E$ s) in the different combinations of attention and practice conditions. DA, divided attention.

the effect of compatibility was significant $[F(1,46)=$ $\left.7.69, M S_{\mathrm{e}}=1.55, p<.01\right]$, where recall was higher in the compatible than in the noncompatible condition. An interaction effect was found only between pace control and compatibility, which approached significance $[F(1,46)=$ $\left.3.08, M S_{\mathrm{e}}=1.14, p<.10\right]$, indicating that the effect of compatibility was more pronounced in the experimentercontrolled pace condition than in the participant-controlled pace condition. Interestingly, the effect of pace control was not significant $\left[F(1,46)=0.01, M S_{\mathrm{e}}=1.43\right.$, n.s.), indicating that performance remained the same regardless of whether the concurrent task pace was controlled by the participants or by the experimenter.

In order to investigate the specific effects of the mediating variables of practice, pace control, and compatibility on recall performance under DA at retrieval and to assess their role in the contrasting results reported in the literature and by Rohrer and Pashler (2003), we compared performance in four selected conditions from those reported above (see Figure 2). The first (A) was performance under full attention. The second (B) was performance in the standard DA-at-retrieval condition used thus far in the literature, which employed equal practice with the memory and the concurrent CRT tasks, a compatible stimulusresponse arrangement, and participant-controlled pace. The third (C) was performance under the conditions used by Rohrer and Pashler with a noncompatible stimulusresponse mapping and an experimenter-controlled pace, but with equal practice in both the memory and the concurrent CRT tasks. The fourth (D) was performance on the task used by Rohrer and Pashler with a noncompatible stimulus-response mapping, an experimenter-controlled pace, and concurrent task emphasis practice.

A series of $t$ tests indicated a significant decrease in performance of about $9 \%$ in the standard DA-at-retrieval condition ( $\mathrm{B} ; M=7.02)$, relative to the full-attention condition $(\mathrm{A} ; M=7.75)[t(23)=2.33, p<.05]$, replicating the standard results reported in the literature (e.g., Craik et al., 1996; Naveh-Benjamin, Craik, Gavrilescu, \& Anderson, 2000; Naveh-Benjamin et al., 1998; NavehBenjamin, Craik, Perretta, \& Tonev, 2000). Similarly, performance decreased significantly by $15 \%$ in Condition C $(M=6.50)$, relative to the full-attention condition (A; $M=7.75)[t(23)=3.72, p<.01]$. Also, performance decreased significantly by $26 \%$ in Condition D $(M=5.52)$, relative to the full-attention condition (A) $[t(23)=5.82$, $p<.01$ ], replicating Rohrer and Pashler's (2003) results. Interestingly, performance in Condition $\mathrm{C}$ was significantly better than that in Condition $\mathrm{D}[t(46)=2.04, p<$ $.05]$ but not significantly worse than that in Condition B $[t(23)=1.59$, n.s.; see Figure 2]. The former comparison indicates that the concurrent task emphasis practice regime used by Rohrer and Pashler may have contributed to their results, whereas the latter comparison indicates that the combined effect of noncompatible stimulus-response mapping and experimenter-controlled pace per se did not result in a significant decrement in performance beyond that seen in the standard conditions used to test the effects of DA at retrieval.

The effects of the attention manipulation on mean recall latency can be assessed by an examination of cumulative recall performance over the 60 -sec recall interval. Figure $3 \mathrm{~A}$ presents the accumulation of the total number of words recalled as a function of time (in 5-sec intervals) during the recall period for the three attention conditions. ${ }^{2}$ The common characteristic of all the functions is the decreasing return as time passed, reflecting the fact that the participants recalled words at an increasingly slower rate until they reached an asymptotic level of performance. Visual inspection of the figure also shows that the initial 


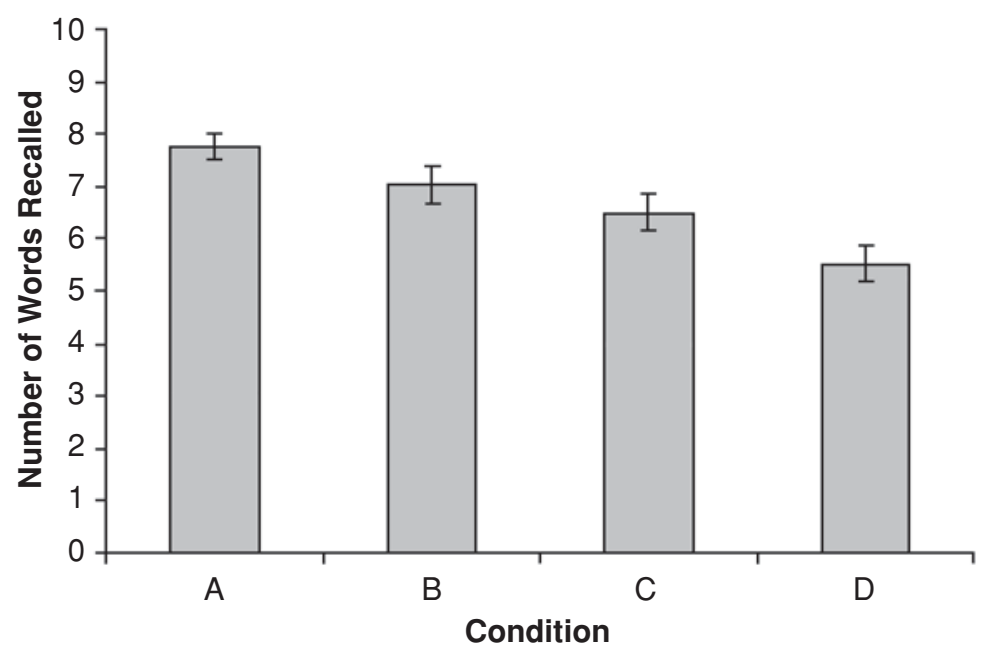

Figure 2. Recall performance $( \pm S E$ s) in four selected conditions: (A) full attention; (B) the standard divided-attention-at-retrieval condition, with equal practice with the memory and the concurrent choice reaction time (CRT) tasks, a compatible stimulus-response arrangement, and a participant-controlled pace; (C) the conditions used by Rohrer and Pashler (2003) with a noncompatible stimulus-response mapping and an experimenter-controlled pace, but with equal practice in both the memory and the concurrent CRT tasks; (D) the conditions used by Rohrer and Pashler with a noncompatible stimulus-response mapping, an experimenter-controlled pace, and concurrent task emphasis practice.

rates of accumulation of the three curves during the first $5 \mathrm{sec}$ differ, with the full-attention condition showing the fastest initial rate, DA at encoding showing somewhat slower rate, and DA at retrieval showing the slowest rate. Furthermore, the recall level seems to asymptote earliest in the DA-at-encoding condition (around $20 \mathrm{sec}$ ), later in the full-attention condition (around $30 \mathrm{sec}$ ), and latest in the DA-at-retrieval condition (around $35 \mathrm{sec}$ ). The initial lower retrieval and later asymptotic level reached in the DA-at-retrieval condition may reflect the inability of the participants to simultaneously initiate the concurrent and the retrieval tasks at the start of the retrieval period. This inability caused an initial delay in recall processes: either delayed search processes or withholding of retrieval responses, due to the processing of the concurrent task. Once the secondary task got underway, the participants were able to concurrently retrieve the information until they exhausted the retrievable words. Cumulative recall performance functions under the different manipulations of the concurrent task showed patterns very similar to those reported above for overall accuracy.

The effects of the attention manipulation on mean recall latency in the different attention conditions can also be assessed using the absolute frequency distribution of recall at each 5-sec interval. Figure 3B shows the effect of the concurrent CRT task on mean recall latency in the different attention conditions. In this figure, the height of each bar represents the number of words recalled in each 5-sec retrieval interval. A visual inspection of these curves reveals that the temporal distribution of responses in the DA-at-encoding condition was similar to that in the baseline condition, with fewer overall responses at each interval. However, this distribution in the DA-at-retrieval condition appears to have shifted to the right, with significantly fewer responses in the first 5 -sec interval, but with either the same number of or more responses in the later intervals, relative to the baseline condition. This distribution (which is similar to the one reported by Rohrer \& Pashler, 2003) indicates that recall performance is slowed down by the concurrent task during retrieval.

\section{Concurrent CRT Task}

Average RTs and average accuracy were calculated for the performance under baseline (single task) and DA (dual task) on the continuous auditory CRT task.

Baseline (single task) CRT. Performance in the baseline CRT task (Table 2) shows that the effects of the manipulations of pace control and stimulus-response compatibility were as expected: RT was longer under the experimenterpaced than under the participant-paced control and was longer also in the noncompatible than in the compatible condition. A two-way ANOVA showed both of these effects to be significant $\left[F(1,46)=35.22, M S_{\mathrm{e}}=25,878\right.$, $p<.01]$ and $\left[F(1,46)=45.62, M S_{\mathrm{e}}=1,789, p<.01\right]$ for the pace and the compatibility manipulations, respectively. In addition, the participants were slowest under the combination of experimenter-paced control and a noncompatible stimulus-response mapping and were fastest under participant-paced control and a compatible stimulusresponse mapping. These results are in line with the expectations (based on Rohrer \& Pashler's, 2003, study) that the former combination makes the concurrent task more 

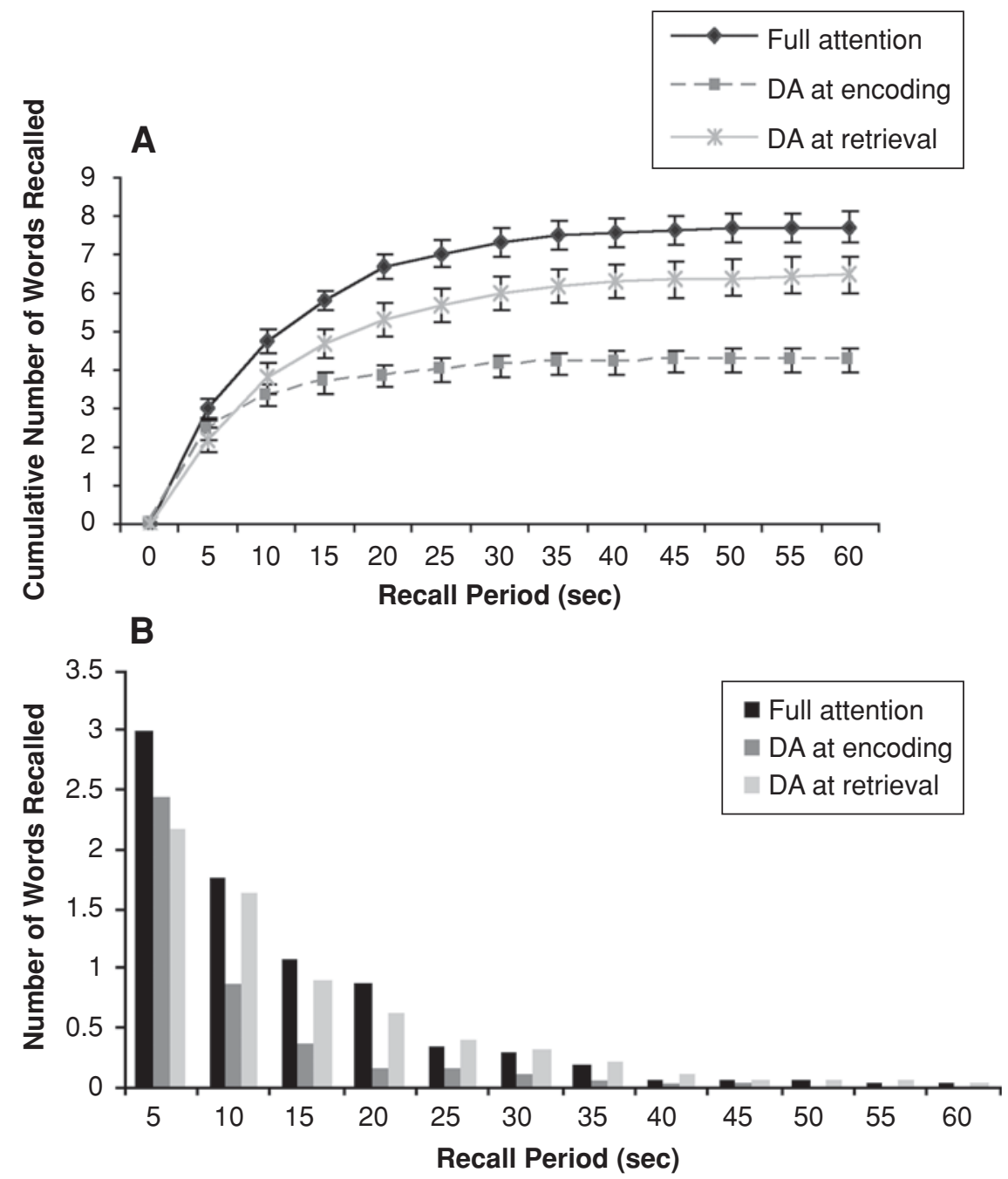

Figure 3. Recall performance. (A) Cumulative recall curves $( \pm S E s)$ as a function of time elapsed during the recall period for the different attention conditions. (B) Recall latency distributions as a function of the different 5 -sec intervals during the recall period for the different attention conditions. DA, divided attention.

demanding and increases its temporal density. Similar patterns were obtained when accuracy of the secondary task was assessed (see Table 2). Note that for the participantpaced control condition, the values in Table 2 reflect the actual ISI, since the participant's given response initiated the appearance of the next concurrent task stimulus. For the experimenter-paced control condition, these values reflect stimulus-response intervals. The actual average ISI for this condition was $877 \mathrm{msec}$. Although this average ISI was longer in the experimenter-paced than in the participant-paced single-task condition, the former task was more difficult for the participants, as is reflected by the longer stimulusresponse interval; the participants had less flexibility in having to respond before the appearance of the next stimuli, something they did not have to do in the latter task, where they could themselves decide when to respond.
CRT as a function of attention and practice. The data presented in Figure 4 allow us to assess whether the manipulation of attention was successful and whether it was affected by the different practice conditions. The figure indicates that CRT performance on the concurrent task under full attention (average baseline condition) was superior to that under the DA conditions and that DA at retrieval resulted in poorer concurrent task performance than did DA at encoding. The results also show that performance on the concurrent task was faster in the concurrent task emphasis practice condition than in the equal practice condition, and this finding was especially true for the DA conditions.

The patterns above were supported by a two-way ANOVA with attention and practice as factors, which showed a significant effect of attention $[F(2,92)=$ 
Table 2

Means and Standard Deviations for Response Time (RT, in Milliseconds) and Accuracy of Performance (Percentage Correct) in the Baseline Concurrent Choice RT Task in Each of the Conditions

\begin{tabular}{|c|c|c|c|c|c|}
\hline \multirow[b]{2}{*}{ Pace } & \multirow{2}{*}{$\begin{array}{l}\text { Stimulus-Response } \\
\text { Mapping }\end{array}$} & \multicolumn{2}{|c|}{ RT } & \multicolumn{2}{|c|}{ Accuracy } \\
\hline & & $M$ & $S D$ & $M$ & $S D$ \\
\hline \multirow{3}{*}{ Experimenter controlled } & Equal Emphasis & & & & \\
\hline & Compatible & 867 & 172 & 93.8 & 7.4 \\
\hline & Noncompatible & 873 & 177 & 90.0 & 10.2 \\
\hline \multirow[t]{2}{*}{ Participant controlled } & Compatible & 666 & 101 & 95.1 & 7.6 \\
\hline & Noncompatible & 699 & 118 & 95.5 & 4.6 \\
\hline \multicolumn{6}{|c|}{ Concurrent Task Emphasis } \\
\hline \multirow[t]{2}{*}{ Experimenter controlled } & Compatible & 765 & 124 & 92.9 & 6.7 \\
\hline & Noncompatible & 791 & 131 & 88.6 & 6.2 \\
\hline \multirow[t]{2}{*}{ Participant controlled } & Compatible & 640 & 124 & 97.8 & 1.3 \\
\hline & Noncompatible & 741 & 124 & 98.4 & 1.2 \\
\hline
\end{tabular}

63.42, $\left.M S_{\mathrm{e}}=26,910, p<.01\right]$. A comparison of CRT baseline $(M=755 \mathrm{msec}, S D=101)$ and DA at encoding $(M=823 \mathrm{msec}, S D=134)$ showed a significant difference $\left[F(1,46)=12.05, M S_{\mathrm{e}}=2,208, p<.01\right]$, as did a comparison of full attention and DA at retrieval $(M=$ $1,110 \mathrm{msec}, S D=340)\left[F(1,46)=22.13, M S_{\mathrm{e}}=40,590\right.$, $p<.01]$. The comparison of DA at encoding and DA at retrieval was also significant $\left[F(1,46)=16.23, M S_{\mathrm{e}}=\right.$ $37,929, p<.01]$. These results replicate those reported in the literature and in Craik et al. (1996) and Naveh-Benjamin et al. (1998): DA at retrieval resulted in a larger increase in concurrent task performance (a 50\% increase from the baseline condition) than did DA at encoding (an 11\% increase).
The ANOVA also showed an effect of practice $\left[F(1,46)=5.02, M S_{\mathrm{e}}=86,070, p<.05\right]$, where CRT performance was better under concurrent task emphasis practice $(M=841 \mathrm{msec})$ than under equal practice $(M=$ $951 \mathrm{msec})\left[F(1,46)=5.03, M S_{\mathrm{e}}=86,070, p<.05\right]$. Finally, the interaction of attention and practice was significant $\left[F(2,92)=3.21, M S_{\mathrm{e}}=26,910, p<.05\right]$. The source of this interaction was the lack of an effect of practice on performance in the single task $\left[F(1,46)=1.83, M S_{\mathrm{e}}=\right.$ 11,339, n.s.] and the significant effects of practice under DA during encoding $\left[F(1,46)=4.45, M S_{\mathrm{e}}=18,055, p<\right.$ $.05]$ and during retrieval $\left[F(1,46)=4.56, M S_{\mathrm{e}}=110,493\right.$, $p<.05$; see Figure 4]. These results attest to the success of the practice manipulation, which resulted in a shorter

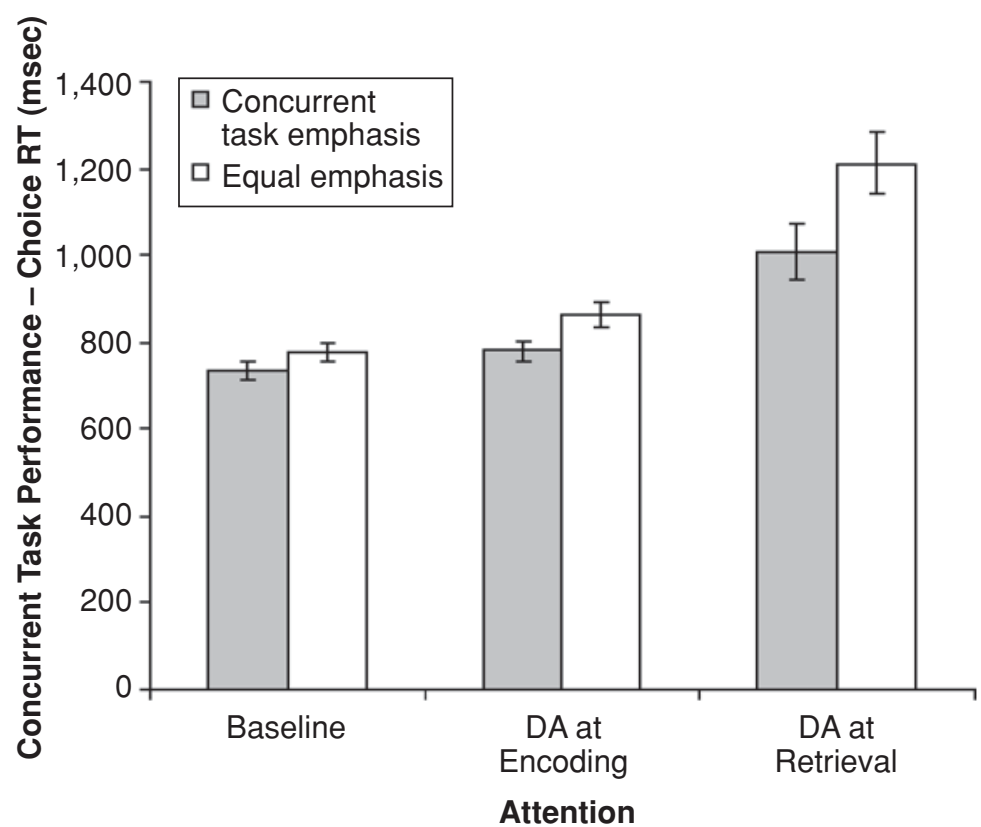

Figure 4. Choice reaction time performance $( \pm S E s)$ in the concurrent task in the different combinations of attention and practice conditions. DA, divided attention. 
Table 3

Means and Standard Deviations for the Attentional Cost (in Milliseconds) Associated With Each of the Conditions

\begin{tabular}{|c|c|c|c|c|c|c|c|c|}
\hline \multirow[b]{3}{*}{ Attention } & \multicolumn{4}{|c|}{ Experimenter-Controlled Pace } & \multicolumn{4}{|c|}{ Participant-Controlled Pace } \\
\hline & \multicolumn{2}{|c|}{ Compatible } & \multicolumn{2}{|c|}{ Noncompatible } & \multicolumn{2}{|c|}{ Compatible } & \multicolumn{2}{|c|}{ Noncompatible } \\
\hline & $M$ & $S D$ & $M$ & $S D$ & $M$ & $S D$ & $M$ & $S D$ \\
\hline \multicolumn{9}{|c|}{ Equal Emphasis } \\
\hline Divided at encoding & 32 & 90 & 22 & 88 & 281 & 215 & 275 & 194 \\
\hline Divided at retrieval & 178 & 272 & 218 & 437 & 616 & 476 & 733 & 516 \\
\hline \multicolumn{9}{|c|}{ Concurrent Task Emphasis } \\
\hline Divided at encoding & 32 & 49 & 3 & 86 & 211 & 107 & 130 & 89 \\
\hline Divided at retrieval & 107 & 165 & 65 & 81 & 483 & 421 & 437 & 484 \\
\hline
\end{tabular}

CRT when the practice phase was devoted mostly to the concurrent task than when it was devoted equally to the concurrent and the memory tasks.

Attentional costs assessed by the CRT. To assess the attentional costs associated with encoding and retrieval, we subtracted, for each participant, the CRT in the baseline performance of a given condition from the CRT performance in the comparable DA condition. These attentional cost scores were averaged for all the participants in every given condition and are presented in Table 3. A four-way ANOVA was conducted with attention, distribution of practice, stimulus-response compatibility, and pace control as the four independent variables and CRT performance as the dependent variable. The analysis showed the effect of attention to be significant $[F(1,46)=$ 33.20, $\left.M S_{\mathrm{e}}=154,948, p<.01\right]$, where attentional costs were higher in the DA-at-retrieval $(M=321 \mathrm{msec})$ than in the DA-at-encoding $(M=126 \mathrm{msec})$ condition. The effect of practice was also significant $[F(1,46)=5.58$, $\left.M S_{\mathrm{e}}=211,085, p<.05\right]$, showing that attentional costs were higher under equal practice $(M=296 \mathrm{msec})$ than under secondary task emphasis practice $(M=185 \mathrm{msec})$ condition. In addition, the effect of pace was significant $\left[F(1,46)=97.53, M S_{\mathrm{e}}=97,145, p<.01\right]$, indicating that concurrent task costs were higher for participantcontrolled pace $(M=398 \mathrm{msec})$ than for experimentercontrolled pace $(M=95 \mathrm{msec})$. The only interaction effect that proved significant was that between attention and pace $\left[F(1,46)=15.66, M S_{\mathrm{e}}=76,313, p<.01\right]$, indicating that attentional costs were larger at retrieval than at encoding in the participant-controlled pace condition, relative to the costs in the experimenter-paced one (see Table 3).

In order to directly assess the role of the mediating variables of practice, pace control, and compatibility in producing different patterns of attentional costs during retrieval, we have compared three selected conditions from those reported above (using the same notation as that used above in the free recall analysis). The first (B) was performance in the standard DA-at-retrieval condition used so far in the literature, which included similar practice with the memory and the concurrent CRT tasks, a com- patible stimulus-response arrangement, and a participantcontrolled pace. The second (C) was performance under the conditions used by Rohrer and Pashler (2003) with a noncompatible stimulus-response and an experimentercontrolled pace, but with equal practice in both the memory and the concurrent CRT tasks. The third condition (D) was the one used by Rohrer and Pashler with a noncompatible stimulus-response, an experimenter-controlled pace, and concurrent task emphasis practice.

A series of $t$ tests indicated a significant decrease in concurrent task costs in Condition C $(M=218 \mathrm{msec})$ $[t(23)=4.13, p<.01]$, relative to the standard DA-atretrieval condition (B; $M=616 \mathrm{msec})$, and a further significant decrease in these costs in Condition D $(M=$ $65 \mathrm{msec}$ ), relative to that in Condition C $[t(46)=1.69$, $p<.05$, unidirectional test]. The former comparison indicates that the participant-controlled pace in the standard DA-at-retrieval conditions that appear in the literature actually results in larger concurrent task costs than when an experimenter-controlled pace is used. Participants possibly delay their responses to the concurrent task when they have control over its pace, in order to protect the retrieval process. The latter comparison indicates that the concurrent task emphasis practice regime used by Rohrer and Pashler (2003) may have contributed to the improved performance of the participants on the concurrent task under DA conditions.

\section{DISCUSSION}

This experiment was carried out with the intention of testing and clarifying several issues related to the effects of DA at retrieval on memory performance. Until recently, almost all studies in which standard memory accuracy measures and, in particular, free recall tasks have been used have shown only a modest decrease in memory performance under DA during retrieval. However, this could have been due to characteristics of the concurrent task employed, which have made it not demanding enough. For example, Rohrer and Pashler (2003) have recently shown much larger effects (an approximate $25 \%$ reduction) in free recall under DA-at-retrieval conditions, relative to perfor- 
mance under full attention, when the temporal density of the concurrent task was increased by using a noncompatible stimulus-response mapping and an experimentercontrolled pace for the concurrent task.

The first question investigated in the present study was whether systematically manipulating the difficulty of the secondary task by contrasting conditions of relatively high and low temporal density would have an effect on memory retrieval. We investigated whether retrieval is affected significantly in the case of high temporal density, as was shown by Rohrer and Pashler (2003), but only mildly in the case of lower temporal density, in line with several studies in the literature (e.g., Baddeley et al., 1984; Craik et al., 1996; Naveh-Benjamin, Craik, Gavrilescu, \& Anderson, 2000; Naveh-Benjamin et al., 1998; Naveh-Benjamin, Craik, Perretta, \& Tonev, 2000). We used a design that allowed the assessment of the standard results reported in the literature, as well as those reported by Rohrer and Pashler. The results showed that when the concurrent task involved a compatible stimulus-response mapping and its pace was controlled by the participant, combined with a standard practice regime with equal emphasis on both tasks, the effects of DA at retrieval on memory accuracy were minimal, with only a $9 \%$ decrease in free recall, relative to a full-attention condition. This result replicates those reported in previous studies, which have shown a reduction of about $10 \%$ in performance under DA at retrieval (e.g., Craik et al., 1996; see Figure 2, Condition B). However, when the concurrent task involved a noncompatible stimulus-response mapping, its pace was controlled by the experimenter, and the practice regime emphasized the concurrent task, the results showed a relatively large drop in retrieval accuracy (similar to those reported by Rohrer \& Pashler, 2003). As is shown in Figure 2, free recall performance in Condition D dropped by $26 \%$, relative to the full-attention condition (close to the $25 \%$ decrease reported by Rohrer \& Pashler, 2003). In addition, the cumulative recall patterns obtained in the present experiment seem very similar to Rohrer and Pashler's results, both for the full-attention and for the DA-at-retrieval conditions (Figure 3). Furthermore, performance on the concurrent task in Condition $\mathrm{D}$ in the present experiment slowed down by $65 \mathrm{msec}$, relative to the baseline condition, when performed simultaneously with recall, close to the 36 msec reported in Rohrer and Pashler's study. This similarity between our results and those reported by Rohrer and Pashler suggests that although we did not use conditions that provided a literal replication, we were able to capture the essence of their manipulations.

Second, we evaluated characteristics of the concurrent task that significantly affected free recall performance by independently manipulating the compatibility of the stimulus-response mapping and the control of the pace of the concurrent task. The results show that a concurrent task that employs noncompatible stimulus-response mapping negatively affects a simultaneous free recall activity. However, whether the concurrent task pace was controlled by the experimenter or by the participant did not seem to have any effect on recall performance. The results pertaining to the differential effects of compatibility and pace on DA during retrieval do not appear to be due to the failure of the pace manipulation; performance in the baseline conditions of the concurrent task clearly slowed down significantly under conditions of experimenter-controlled pace and when stimulus-response mapping was noncompatible (see Table 2).

The failure of the control of the concurrent task pace to affect memory performance is in line with the results reported by Craik, Naveh-Benjamin, Ishaik, and Anderson (2000), which showed memory retrieval accuracy under DA conditions not to be affected by whether the appearance of the stimuli in the memory task was participant or experimenter controlled. Hicks and Marsh (2000), using a recognition paradigm, also showed significant effects of DA at retrieval when several experimenter-controlled pace concurrent tasks were used. The results obtained in our study indicate that the effects shown by Hicks and Marsh might have been due to factors other than the secondary task's being controlled by the experimenter.

Third, we also assessed here the effects of another feature of the dual task that might affect the pattern of results - namely, the practice given to the memory and the secondary tasks. We manipulated the practice regime, using either a heavy emphasis on performance in the concurrent task (as in Rohrer \& Pashler, 2003) or a more standard method, which enables similar distribution of practice on the memory and the concurrent tasks, as well as on performing both together. The results indicate that when a standard practice schedule is used (even with manipulations that create high temporal density), the effects of DA at retrieval are significantly smaller than when a heavy emphasis on practice in the concurrent task is used, with performance under DA at retrieval in the former dropping only by $15 \%$, relative to the full-attention condition (see Condition C in Figure 2). This drop is significantly smaller than the $25 \%$ drop reported by Rohrer and Pashler, who employed a practice phase that emphasized the concurrent task. Furthermore, this drop is not significantly different from the $9 \%$ drop shown in the standard DA-atretrieval condition. The concurrent task costs showed a complementary pattern and were significantly smaller in the concurrent task emphasis condition than in the equal practice one, suggesting that the participants allocated fewer resources to the memory task in the former case. Overall, these results indicate that the practice regime used for the memory task and the concurrent task may play a role in participants' performance on both tasks.

Fourth, the present results show that even under conditions that create high temporal density, the asymmetry in the effects of DA at encoding and retrieval on memory accuracy is maintained. The results show that in contrast to the effects of DA at retrieval, which ranged from $9 \%$ to $26 \%$, depending on the condition, the effects of DA at encoding were much larger, ranging from $46 \%$ to $59 \%$. Likewise, concurrent task performance also revealed the asymmetry reported in several previous studies (e.g., 
Craik et al., 1996; Naveh-Benjamin, Craik, Gavrilescu, \& Anderson, 2000; Naveh-Benjamin et al., 1998; NavehBenjamin, Craik, Perretta, \& Tonev, 2000), with concurrent task costs being significantly larger during retrieval than during encoding.

Altogether, the results of our present study show that the effects of DA during retrieval vary and may depend on the practice regime and, to some degree, on the temporal density of the concurrent task. In particular, when the mapping of a stimulus to a response in the concurrent task is not compatible, the extra attentional effort required for the response selection in the concurrent task seems to affect concurrent memory retrieval (see Rohrer \& Pashler, 2003). Interestingly, the control that participants have over the pacing of the stimuli appearance during the concurrent task does not appear to affect concurrent memory retrieval; memory retrieval does not appear to suffer even when the participants have no control over the pace in which the concurrent task is presented. Furthermore, despite the fact that with the experimenter-controlled concurrent task pace, participants allocate less attention to the retrieval task than they do with the participant-controlled pace (as reflected in the smaller concurrent task costs in the former), retrieval accuracy performance does not seem to suffer under these conditions. All of these findings seem to indicate that although retrieval processes might not be automatic, in that they require substantial attentional resources, they may nevertheless be more obligatory or protected and less prone to interference, at least relative to encoding processes.

The present results do support the claim that the use of a compatible stimulus-response mapping may obscure the effects of concurrent task on memory retrieval (Rohrer $\&$ Pashler, 2003). In this sense, the manipulation of the compatibility of the concurrent task seems to be only one of a few manipulations shown so far to affect the accuracy of memory retrieval. Several other manipulations used to date, including the amount of information to be remembered, word frequency, and the control of the pace of the memory or the concurrent task, seem to mostly affect concurrent task, but not memory task, accuracy.

Note that the relative resilience of retrieval to the effects of interference in this study characterized retrieval accuracy, but not retrieval latency, since the latter slowed down especially during the first few seconds of the free recall period. This pattern, which has been reported by other researchers (e.g., Baddeley et al., 1984; Naveh-Benjamin \& Guez, 2000; but see Naveh-Benjamin, Craik, Guez, \& Krueger, 2005), indicates that participants are able, to some degree, to appropriately search for and access target information under interference but that these processes are slowed down significantly.

One factor thus far clearly shown to affect memory retrieval accuracy is the similarity of the materials used in the concurrent task to those used in the retrieval task, since retrieval from memory suffers under conditions of such high similarity (Fernandes \& Moscovitch, 2000,
2002, 2003; Park et al., 1989). In this sense, an overall evaluation of the literature appears to indicate fundamental differences between encoding and retrieval processes. Encoding processes appear to be affected by any type of manipulation that makes the concurrent task more difficult. Retrieval processes, in contrast, appear, in many cases, to be immune to such effects, at least with respect to retrieval accuracy, but this protection requires substantial resources. There are cases in which retrieval processes are clearly interrupted, mostly when the same processing/ representations are required by both the concurrent and the memory tasks.

\section{REFERENCES}

Anderson, N. D., Craik, F. I. M., \& Naveh-Benjamin, M. (1998). The attentional demands of encoding and retrieval in younger and older adults: I. Evidence from divided attention costs. Psychology \& Aging, 13, 405-423.

Baddeley, A. [D.], Lewis, V., Eldridge, M., \& Thomson, N. (1984). Attention and retrieval from long-term memory. Journal of Experimental Psychology: General, 113, 518-540.

Craik, F. I. M., Govoni, R., Naveh-Benjamin, M., \& Anderson, N. D. (1996). The effects of divided attention on encoding and retrieval processes in human memory. Journal of Experimental Psychology: General, 125, 159-180.

Craik, F. I. M., Naveh-Benjamin, M., \& Anderson, N. D. (1998). Encoding and retrieval processes: Similarities and differences. In M. A. Conway, S. E. Gathercole, \& C. Cornoldi (Eds.), Theories of memory II (pp. 61-86). Mahwah, NJ: Erlbaum.

Craik, F. I. M., Naveh-Benjamin, M., Ishaik, G., \& Anderson, N. D. (2000). Divided attention during encoding and retrieval: Differential control effects? Journal of Experimental Psychology: Learning, Memory, \& Cognition, 26, 1744-1749.

Fernandes, M. A., \& Moscovitch, M. (2000). Divided attention and memory: Evidence of substantial interference effects at retrieval and encoding. Journal of Experimental Psychology: General, 129, 155-176.

FERnANDES, M. A., \& Moscovitch, M. (2002). Factors modulating the effect of divided attention during retrieval of words. Memory \& Cognition, 30, 731-744.

Fernandes, M. A., \& Moscovitch, M. (2003). Interference effects from divided attention during retrieval in younger and older adults. Psychology \& Aging, 18, 219-230.

HicKs, J. L., \& MARSH, R. L. (2000). Toward specifying the attentional demands of recognition memory. Journal of Experimental Psychology: Learning, Memory, \& Cognition, 26, 1483-1498.

JACOBY, L. L. (1991). A process dissociation framework: Separating automatic from intentional uses of memory. Journal of Memory \& Language, 30, 513-541.

Johnston, W. A., Greenberg, S. N., Fisher, R. P., \& Martin, D. W. (1970). Divided attention: A vehicle for monitoring memory processes. Journal of Experimental Psychology, 83, 164-171.

Kerr, B. (1973). Processing demands during mental operations. Memory \& Cognition, 1, 401-412.

Martin, D. W. (1970). Residual processing capacity during verbal organization in memory. Journal of Verbal Learning \& Verbal Behavior 9, 391-397

Naveh-Benjamin, M., Craik, F. I. M., Gavrilescu, D., \& Anderson, N. D. (2000). Asymmetry between encoding and retrieval processes: Evidence from divided attention and a calibration analysis. Memory \& Cognition, 28, 965-976.

Naveh-Benjamin, M., Craik, F. I. M., Guez, J., \& Dori, H. (1998). Effects of divided attention on encoding and retrieval processes in human memory: Further support for an asymmetry. Journal of Experimental Psychology: Learning, Memory, \& Cognition, 24, 1091-1104.

Naveh-Benjamin, M., Craik, F. I. M., Guez, J., \& Krueger, S. (2005). Divided attention in younger and older adults: Effects of strategy and 
relatedness on memory performance and secondary task costs. Journal of Experimental Psychology: Learning, Memory, \& Cognition, 31, 520-537.

Naveh-Benjamin, M., Craik, F. I. M., Perretta, J. G., \& Tonev, S. T. (2000). The effects of divided attention on encoding and retrieval processes: The resiliency of retrieval processes. Quarterly Journal of Experimental Psychology, 53A, 609-625.

Naveh-Benjamin, M., \& Guez, Y. (2000). The effects of divided attention on encoding and retrieval processes: Assessment of attentional costs and a componential analysis. Journal of Experimental Psychology: Learning, Memory, \& Cognition, 26, 1461-1482.

Park, D. C., Smith, A. D., Dudley, W. N., \& Lafronza, V. N. (1989). Effects of age and a divided attention task presented during encoding and retrieval on memory. Journal of Experimental Psychology: Learning, Memory, \& Cognition, 15, 1185-1191.

PASHLER, H. (1998). The psychology of attention. Cambridge, MA: MIT Press.

Rohrer, D., \& PASHLER, H. E. (2003). Concurrent task effects on memory retrieval. Psychonomic Bulletin \& Review, 10, 96-103.

Troyer, A. K., Winocur, G., Craik, F. I. M., \& Moscovitch, M. (1999). Source memory and divided attention: Reciprocal costs to primary and secondary tasks. Neuropsychology, 13, 467-474.

\section{NOTES}

1. Since compatibility was manipulated within participants, several pilot participants who were tested with $\mathrm{A}, \mathrm{B}$, and $\mathrm{C}$ and with $\mathrm{B}, \mathrm{A}$, and $\mathrm{C}$ for the compatible and the noncompatible conditions, respectively, reported a confusion in changing their mappings from one trial to the next. To avoid such confusion we used R, L, and P for the noncompatible condition. Pilot data indicated no differences in CRT performance between the R, $\mathrm{L}$, and $\mathrm{P}$ and the $\mathrm{B}, \mathrm{A}$, and $\mathrm{C}[M=788$ and $779 \mathrm{msec}$, respectively; $t(11)=0.71$, n.s.].

2. The distribution of cumulative recall in each $5 \mathrm{sec}$ might be tilted by about $1 \mathrm{sec}$ toward showing responses later than when they actually occurred, since, as was described in the Method section, recall responses were made orally by the participants and were then entered into the computer by the experimenter, on the average, $1 \mathrm{sec}$ later. This, however, was consistent throughout the different conditions and, hence, did not affect the overall pattern of cumulative recall.

(Manuscript received July 29, 2004; revision accepted for publication January 7, 2005.) 